\title{
Gait Analysis of Pedestrians with the Aim of Detecting Disabled People
}

\author{
Amir Mukhtar ${ }^{\mathrm{a}^{*}}$, Michael J. Cree $^{\mathrm{b}}$, Jonathan B. Scott ${ }^{\mathrm{c}}$ and Lee Streeter ${ }^{\mathrm{d}}$ \\ School of Engineering, University of Waikato, Hamilton, New Zealand \\ aam301@students.waikato.ac.nz, bmichael.cree@waikato.ac.nz, cjonathan.scott@waikato.ac.nz, \\ dee.streeter@waikato.ac.nz
}

Keywords: gait, skeletonize, disable, vision

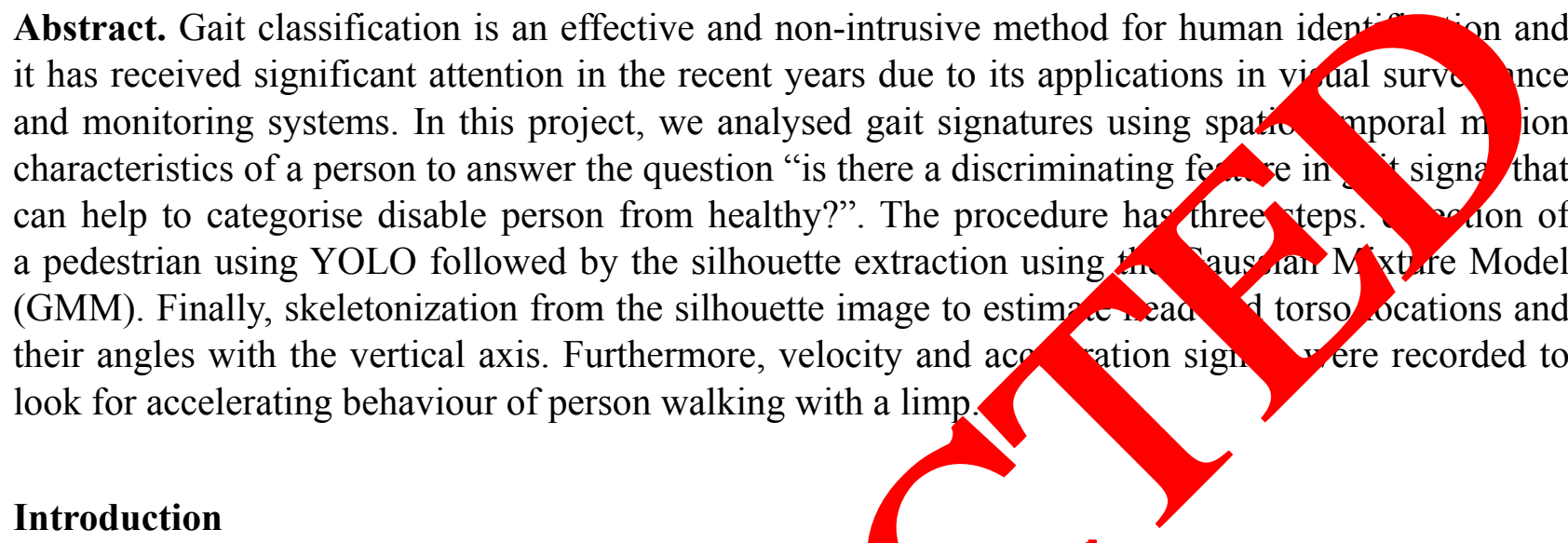

This research aims to investigate computer vision and $p$ rn recog dition techniques for identifying physically disabled people appearing in surveil footag o challenging task since outdoor scenarios with background clutter, illumination Dirm camera viewing angle and camera resolution affect the quality of information sensed. Sce e obta ed from a surveillance video are usually low resolution which adds to the com ${ }^{n} \mathrm{y}$ of the roblem. Video surveillance technology is gaining popularity these days due to its loy ost, e e of inst yation, freedom from interference, and its ability to capture detailed information ovelopments in computer vision, pattern recognition and machine learning h ve furthe creased the usefulness of optical sensors. Advancements in optical sensors and rapid 1 ing costs o gh resolution cameras make them a preferred choice for an automated detection stem ign. We focus on gait signatures of a person walking to investigate whether gait can us cury distingu. Kealthy and disable persons. This involves pedestrian detection followed by extr etion o he gait signal and its analysis. Skeletonization approach is computationally cheap and summa the int nal motion/gait of moving pedestrian without a prior human model.

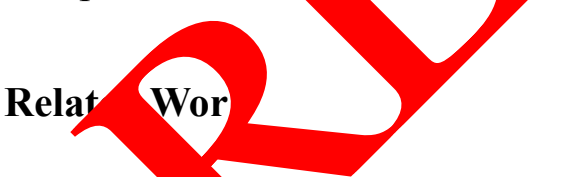

Vision bas lisable person detection involves extraction of moving objects (silhouettes) and their gait analysis categorizing them into healthy or disable. Advances in computer vision lack such a system due to its limited applications. However, a great deal of research has been conducted on vision based pedestrian detection [1], human behaviour detection [2,3] and gait recognition [4]. Therefore, the related literature (which may contribute to develop a gait based disable person detector) is divided into two parts,

\section{Motion Detection and Silhouette Extraction}

Background subtraction is a quick way of localizing moving objects in videos by a static camera and it is often the first step of a multi-stage computer vision system [5] (car tracking, pedestrian detection, wild-life monitoring, etc.). It assumes video sequence comprising of a static background in front of which moving objects (in distinct colours) are extracted. Some commonly used background modelling techniques are inter-frame change [6], GMM [7] and optical flow [8]. Each approach has 
its advantages and disadvantages [9]. We picked GMM based for the system because it is good in handling multi-modal background scenarios.

Background modelling techniques result in silhouettes estimation of moving objects in a video sequence. Most gait analysis procedures $[10,11]$ rely on pre-detection of silhouettes to extract motion signals. While some approaches [12] take bounding boxes containing pedestrians. Pedestrian detection can be performed in two sequential steps: extracting candidate regions that are potentially covered by human objects and classifying the regions as human or non-human. Background subtraction is the simplest way to segregate moving objects and then declaring those as candidate regions. Another common approach is to use a sliding window method in which windows are extracted at various scales and positions without any prior knowledge of the size and location of the human object 4 human classifier trained on the features extracted from the image database, labels the regio $s$ as hu. is or otherwise.

Convolutional Neural Networks (CNNs) are currently the most popular hind and and rithms in computer vision for human detection [13, 14]. You Only Look once YYOLQ 1, presented a new approach to object detection in which a single CNN predica un $\mathrm{ng}$ b $\mathrm{res}$ and class probabilities directly from full images in one evaluation. Being a sin seth it can op optimized resulting in real-time processing of input images at 45 frames per se d. At this ion rate (speed), it outperformed other detection methods, including Deformable arts del (DPD/) and R-CNN. We employed this network for the outdoor set of experiments.

\section{Gait Detection and Analysis}

Model free set of approaches $[15,16,17]$ have also en propose $d$ in which gait is comprised of static components (size and shape) and a dynamic com ant of a person which reflects the actual movement. In gait recognition the static feature height, sumength and silhouette bounding box lengths. Dynamic features used are legs and hea any, ith centroid, velocity, acceleration and frequency of body parts contributing to the overall $\mathrm{m}$ - $\mathrm{H}$. Sal ar et al. [18] created the HumanID project which is considered a significant lan in gait cognition. It was reported that the lower $20 \%$ of silhouette, that is, from the knee $f$ va, a lounted $80 \%$ of the recognition. BenAbdelkader et al. [17] extracted the bounding bo of motion after tracking the subject in video steam. These scaled boxes, known is templa were subtracted from each other pixel by pixel (spatially) for all given pairs of tim in ces (tem, ally). In their later study [19], stride length and cadence (walking frequency) yas ropos to illustrate the human motion by segmenting and erecting a bounding box around it. requency of h. Was estimated by noticing the change in size of the bounding box over time. ${ }^{2}$ iyosh Liptoh [20] analysed motion of a human by detecting its silhouette and boundaries to pro a star s leton. Cyclic motion of skeletal segments was extracted to determine human act such wall ang or running. Although their technique is computationally inexpensive and ap icabl o real-r ad surveillance videos, it is restricted to static background and shadow less scenar

Tempo. epresentation approaches model gait signatures by an XYT three dimensional space by accommodati, time as the third dimension complementing the XY axes in the image plane. Niyogi and Adelson [21] formed an image cube by stacking images of a walking sequence (XY) over the time axis. They analysed the XT plane and traced out a unique braided pattern walker's ankle as compared to the linear moving pattern of head. This information was helpful to construct the stick model and to identify a walker in image sequence by gait. In another study [12], gait sequence was decomposed into XT slices to produce a periodic pattern termed Double Helical Signature through an iterative curve embedding algorithm. This representation highlighted body parts for gait recognition, typically in the context of surveillance. This work relies on availability of gait sequences extracted from the surveillance videos before estimating double helical signatures. In an automated approach, getting gait sequence from silhouette mask is a difficult task specially where shadow is accompanied by human movement. 


\section{Motivation}

A competent observer can pick a disable person by appearance (if a visible mobility aid is in use) or by observing gait (in case of limping walk). Apart from the $3 \mathrm{~d}$ information, he/she can also identify a disable person by monitoring the subject's movement in a monocular video of human walk. This study is intended for observing a distinguished feature in human walk which enabled automated detection of disable person from videos. There is a good chance that a time series of body part movement will depict abnormality sensed by human cognition. Following the approach presented by Fujiyoshi \& Lipton [20], we examine temporal behaviour of head and leg positions.

This paper makes two contributions. First, it separates the joint gait signal produced by legs into two each corresponding a leg. In prior work $[20,11]$ a single gait signal was used but cates the individual action of legs. This new set of gait signal better illustrates the cont bution of $s$ in human walking style. Second, we compare gait signals acquired by manual and o vuter as ted approaches for discerning features to detect a disable person.

\section{Automated Approach}

The topic of disability being sensitive, has less training and tes ing lata a rable on internet. We collected surveillance videos in August 2017, which resul' a n few imber of participants since majority of these people tend to stay at home and approxin ately $0.4 \%[22]$ ear on public places (in NZ). Collected videos involved healthy and disable per on movements with the same camera angle and background. The automated task was divided into s ouette extr ction, skeletonization and Gait analysis phases which are explained below.

\section{Silhouette Extraction}

It can be difficult to analyse the nature of hu nany without extracting the silhouette. Since we target system deployment for outdoor environ $\mathrm{n}$-nts, s, a static background assumption may not hold true. Furthermore, motion detec chnique $[7,8]$ incorporate shadows as part of the moving objects which leads to incorrectly eterm ned silho stte mask, therefore, we started with YOLO to localize pedestrians appearing th $\mathrm{O}$ takes a video frame as input and after processing, provides bounding box with fiv edictions: centre of object (x,y), width (w), height (h), and confidence value. Inside $\mathrm{C}$ CNN pe rmed the detection task with its initial convolutional layers extracting feature of $m$ the while fully connected layers predicted output probabilities and coordinates. An ex ample detection YOLO is shown in Fig. 1. The resulting bounding box serves as the region of terest 30 ) to be segmented into human silhouette. Inside this ROI, foreground subtraction was $\mathrm{p}$ med us $\mathrm{g}$ GMM resulting in silhouette mask. Advantage of using YOLO is that it filt $\mathrm{ta} \mathrm{bi}_{\mathrm{s}} \mathrm{c}$, shadow linked with the moving silhouette and reduces the data size for
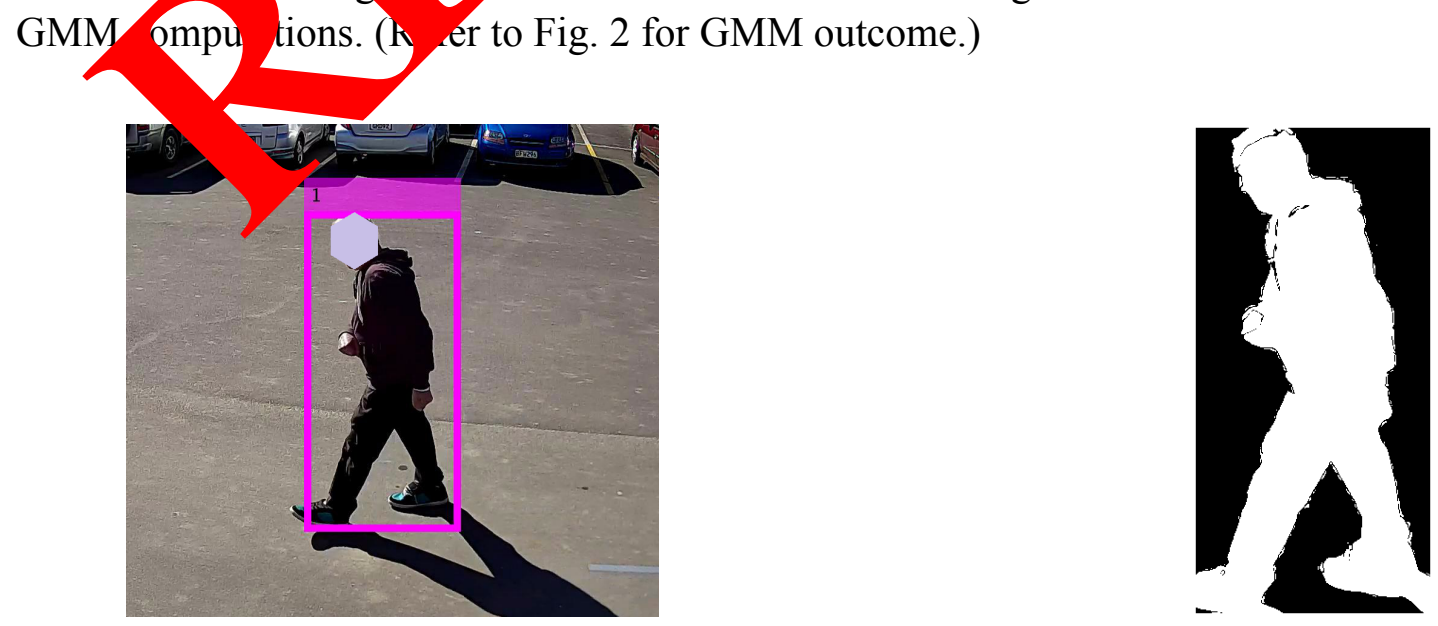

Fig. 1: Pedestrian detection using YOLO

Fig. 2: Pedestrian mask after GMM operation 


\section{Skeletonization and Gait Signal}

To extract the gait signal, we used the approach suggested by Fujiyoshi \& Lipton [20] which assumes a static background with simplified conditions. The difference in our experiment is that YOLO implementation preceded the GMM operation to get the pedestrian mask. After cleaning this mask by morphological operations, its outline was extracted using a border following algorithm (see Fig. 3(a)). The skeleton with two legs and a torso, was constructed by connecting these extremal points to the centroid of silhouette. To analyse the gait, legs and torso angles (Fig. 3(b)) with vertical axis were computed and analysed as time series data. The following gait features in temporal domain were observed in this research:

- Legs velocity and acceleration

- Head velocity and acceleration

- Legs and Head angle

\section{Signal Split}

It has been observed that the leg angle signal is contributed by bo 10, an alte hate fashion based on leading and trailing status. This makes it less reliable for ex nining ind dual eg movements over time. Moreover, locations/positions of legs can be estimated on ve fragme che leg signal into its individual signals. Since monocular camera lacks stereo in o hation, legs are referred to as leg 1 and leg 2 instead of left and right. These splintered signe s have improvo dicators for anomalous walking behaviour. The process of signal split (for the $t$ get moving from right to left of the screen) is summarised as,

1. Extract leg angle data $\theta_{1}$ and $\theta_{2}$ [20]. Here, $\theta_{1}$ and fen leading and trailing leg angles, respectively, both measured from the ver

2. Identify time instants (for $t=t_{n}$, where $n=1,2,3, \ldots$ ) when both legs have angles closest to vertical axis. This occurs at the minima of $\theta_{1}$ so, with $S(t)$ as a logical array namely,

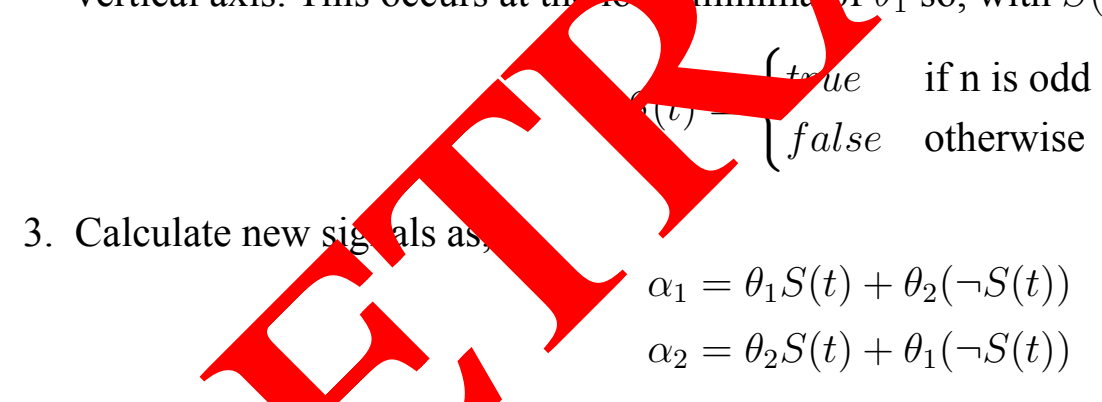

4. Tract-xtrem ints for legs from newly generated signals to form the gait vector.

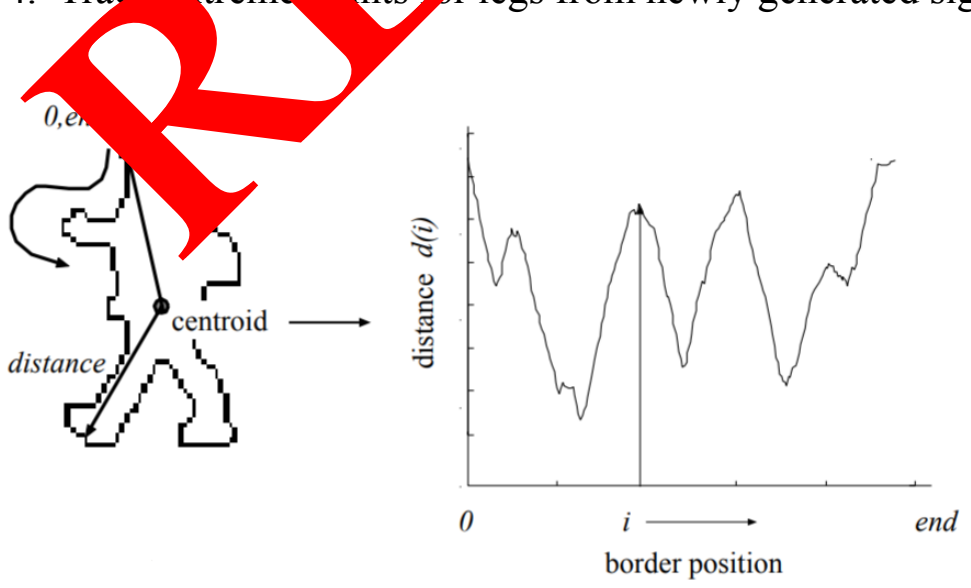

(a) Border Extraction [20]

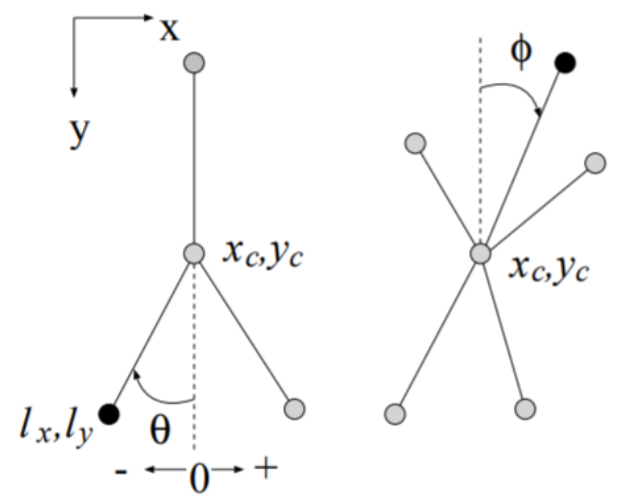

(b) Skeleton Features

Fig. 3: Star Skeletonization 


\section{Experimental Results and Discussion}

\section{Signal Extraction Results}

Signal extraction was tested on indoor data acquired from The Institute of Automation, Chinese Academy of Sciences (CASIA-B) [23] gait dataset. Results (Fig. 4) show the successful operation for retrieving leg signals of a healthy person and has correlation of -0.9556 depicting highly symmetrical movement. Later on, the same experiment was repeated on videos for healthy and disable person movements in an outdoor environment to obtain leg positions with respect to time. Symmetry values of -0.9274 and -0.9174 were obtained for healthy and disabled person movements respectively. The negative sign in correlation is due to movement of legs in opposite directions. Outp were not as smooth as those of indoor case and fluctuations were noted due to inaccuraf es in pec rian localization by YOLO and presence of shadows. This makes leg angles less rem for dete ing abnormal walking behaviour. According to YOLO authors, localization erro ccou for mo e of

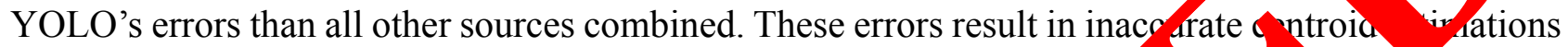
and error propagates in head/legs angle computations.

\section{Automated Results}

In skeletonization scheme, leg angles were estimated using tr go netric fun ons applied on leg and centroid positions predicted by YOLO and GMM foregro nask. mough angular information lacked distinguishing features, it can help us analyse othe monon featuro velocity and acceleration of legs. The fact that lower $20 \%$ portion of human body contributes $80 \%$ in human recognition by gait [18], leg velocity/acceleration may contain use gait inforn ation. Head angle and position could also be investigated for gait signal but our experim reveale that head signal is too sensitive to fluctuations caused by rotations and minor 1 movemo. - arthermore, head is a signal source for its gait signal while, leg data is contributed 2 ou. prone to inaccurate readings compared to the hea d a.

Results for velocity/acceleration an f health and disabled person were generated with automated approach. It was observed toth ets of gro ohs were noisy and it was hard to identify motion patterns for disabled person fro the question that, "is there a discriminating feature in gait signal that can help categoris "sable person from healthy?," remains unanswered. If yes, what caused the failure at ated appi ach for identifying gait feature of a disabled person? This lead us to manually on lent the cation of legs and head locations in video frames over time, and results are reporte $1 \mathrm{n}$ Figs. 5 and

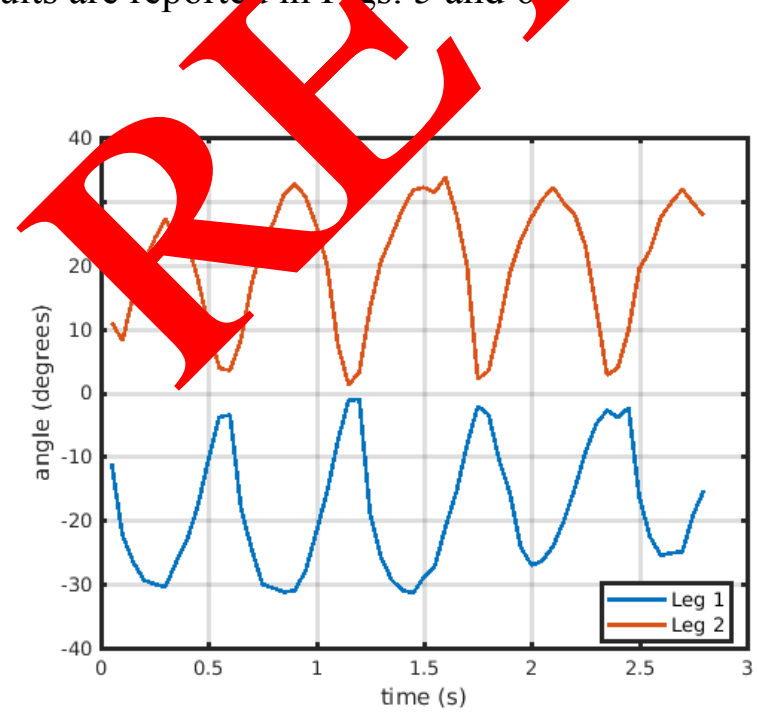

(a) Original signals

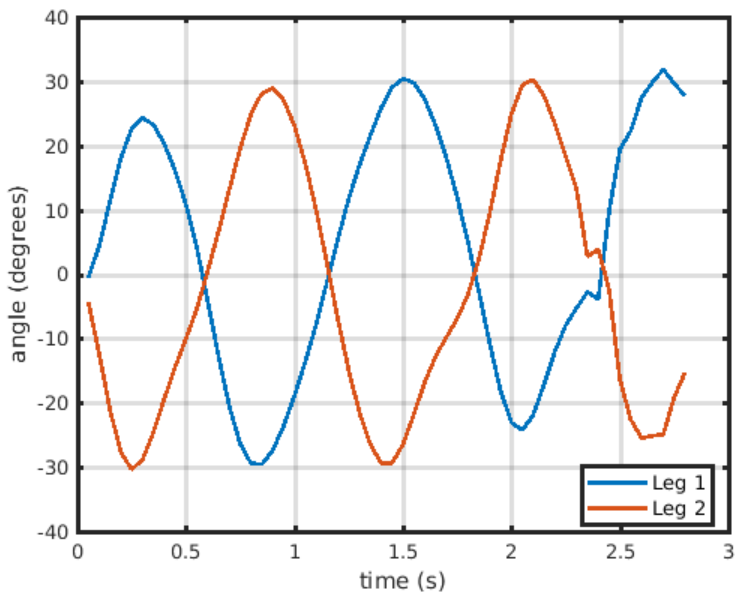

(b) Improved Signals

Fig. 4: Leg signals of Healthy Person in indoor environment 


\section{Manual Results}

We marked locations of legs and head of moving person in video frames and by looking at their plots, there was a clear difference in the graph of the disable person. Graphs for disabled part (leg 2 in figs. 5(b) and 6(b)) clearly produces a signal for leg 2 which is different to that of leg 1 . We considered average values of five peaks shown in Fig. 5 for these calculations. Legs of a healthy person have $1.45 \%$ difference (w.r.t larger value) in peak values while that of disabled person is $47.02 \%$. Similar behaviour is observable in acceleration signals. This shows that Leg 2 of the disabled person is behaving differently than Leg 1 while walking, which means either of these legs is affected and not normal. Head signal was also exploited but it failed to give us encouraging results while centroid of the body is difficult to pick since it requires accurate localization and is sensitive to noise.

\section{Conclusion}

Gait recognition procedures are affected by the variation in environmenta cons aints, wh angle, occlusions, shadows, imperfection in foreground modelling, object nen atton ad silhouette
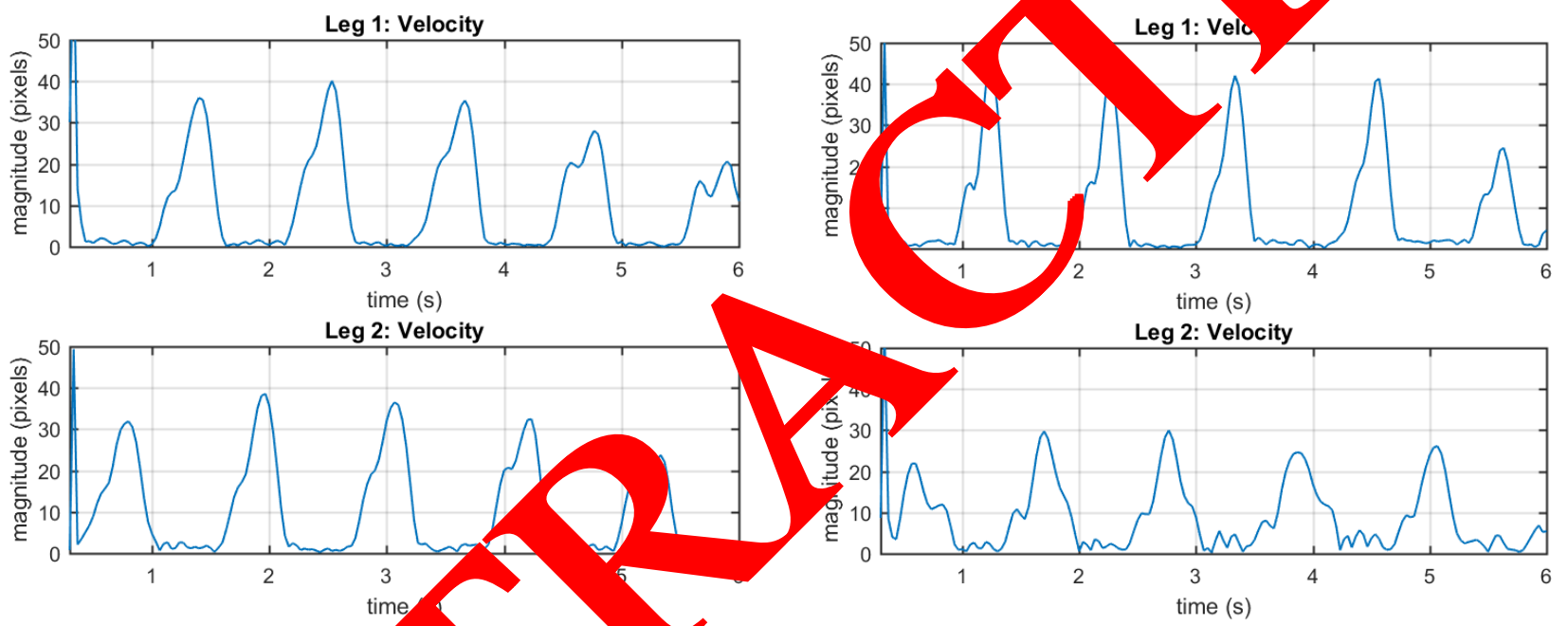

(b) Disabled Person

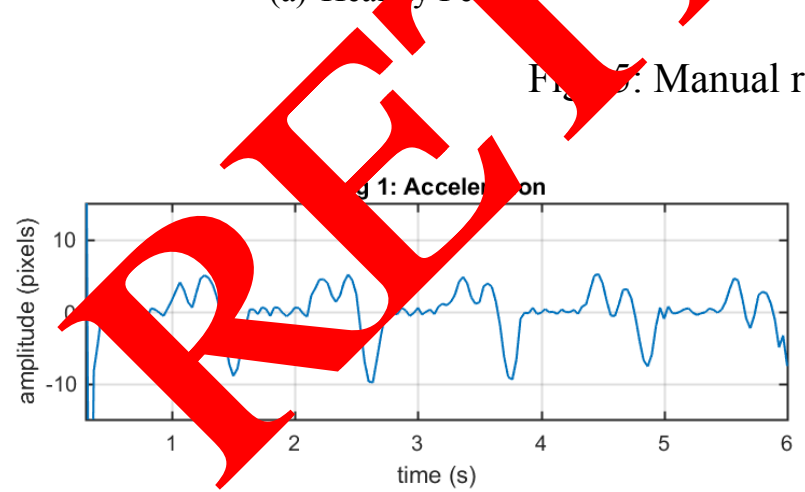

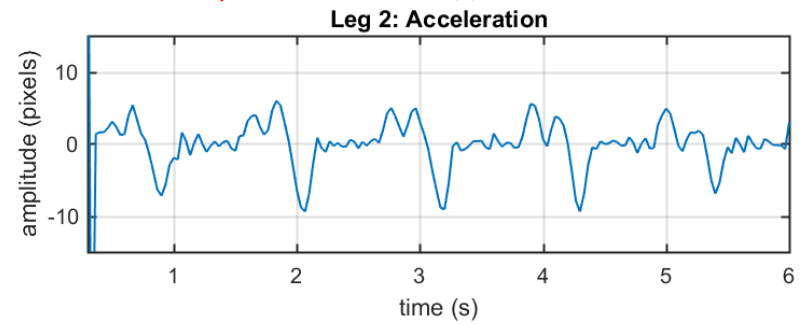

(a) Healthy Person

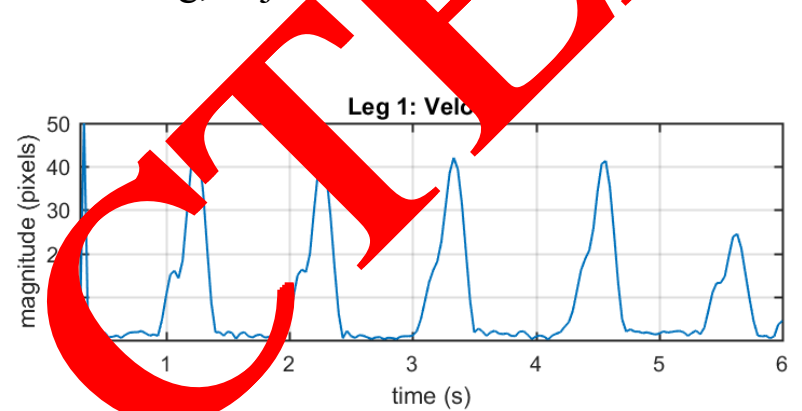

Leg 2: Velocity

lts for velocities
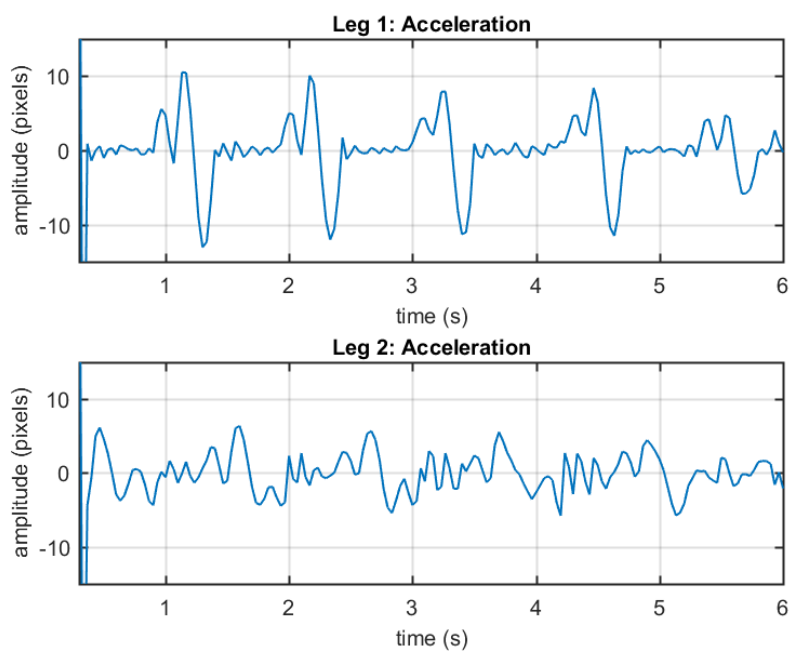

(b) Disabled Person

Fig. 6: Manual results for acceleration 
extraction. We experimented on video data of moving pedestrians to investigate presence of disabled person by automated and manual analysis of head and leg data. The automated system failed to recognise a disabled person from its gait due to imperfection in segmentation, but results from the manual localization suggest that there is enough information in the gait signal to characterize a healthy motion given a set of gait signatures. YOLO's localization error restricts the automated system to show impressive results and problem might be addressed by replacing YOLO with better localized pedestrian detection systems since exact locations of legs and centroid play a key role in shaping a gait signal. Furthermore, work on human joints detection can significantly improve gait signal which may lead to autonomous detection of disabled person. CNN based systems [24] have shown encouraging results in identifying joint locations and connecting them to other body parts of the same person. Such system have tendency to get better results than traditional gait techniques. We shall also be formio CNN based solution to this problem along with identifying people using various mobilit aids.

\section{Acknowledgement}

This study is funded by project TRAD1401 of Callaghan Innovation Ven aland. appreciate Traffic Design Group (TDG) and Stroke Foundation for their supp $c$ in data 4 ctic

\section{References}

[1] Manoranjan Paul, Shah ME Haque, and Subrata C akraborty. Human detection in surveillance videos and its applications-a review. EURASIP urnal on fdvances in Signal Processing, 2013(1):1-16, 2013.

[2] Palwasha Afsar, Paulo Cortez, and Henriqu Jan Automatic visual detection of human behavior: A review from 2000 to 2014. Expert Sy, evo wit, Applications, 42(20):6935-6956, 2015.

[3] Oluwatoyin P Popoola and Ke an m. Vide based abnormal human behavior recognition - a review. Systems, Man, and arne Part Aplications and Reviews, IEEE Transactions on, 42(6):865-878, 201

[4] Tracey K. M. Lee, Mo med Bel atir, and Saeid Sanei. A comprehensive review of past and present vision-ased niques for gait recognition. Multimedia Tools and Applications, 72(3):2833-2\%69,2013.

[5] Dimitios Th is and Ti Ellis. Learning semantic scene models from observing activity in visual anveillà Syst ms, Man, and Cybernetics, Part B: Cybernetics, IEEE Transactions on, 35 s):3, 408,2

[6] Sa a ary, Yasushi Nakata, Toshi Takamori, and Motofumi Hattori. Human detection and lo zation at indoor environment by home robot. In Systems, Man, and Cybernetics, 2000 IEEE Int hational Conference on, volume 2, pages 1360-1365. IEEE.

[7] Chris Stauffer and W Eric L Grimson. Adaptive background mixture models for real-time tracking. In Computer Vision and Pattern Recognition, 1999. IEEE Computer Society Conference on., volume 2. IEEE.

[8] Berthold K.P. Horn and Brian G. Schunck. Determining optical flow. Artificial Intelligence, 17(1):185 - 203, 1981.

[9] Yannick Benezeth, Pierre-Marc Jodoin, Bruno Emile, Hélene Laurent, and Christophe Rosenberger. Comparative study of background subtraction algorithms. Journal of Electronic Imaging, 19(3):033003-033003-12, 2010. 
[10] Imad Rida, Ahmed Bouridane, Gian Luca Marcialis, and Pierluigi Tuveri. Improved human gait recognition. In International Conference on Image Analysis and Processing, pages 119-129. Springer, 2015.

[11] Liang Wang, Tieniu Tan, Huazhong Ning, and Weiming Hu. Silhouette analysis-based gait recognition for human identification. IEEE Transactions on Pattern Analysis and Machine Intelligence, 25(12):1505-1518, 2003.

[12] Y. Ran, Q. Zheng, R. Chellappa, and T. M. Strat. Applications of a simple characterization of human gait in surveillance. IEEE Transactions on Systems, Man, and Cybernetics, Part B (Cybernetics), 40(4):1009-1020, 2010.

[13] Xingyu Zeng, Wanli Ouyang, Meng Wang, and Xiaogang Wang. Deep learnin of scene-sp ific classifier for pedestrian detection. In European Conference on Computer Vision, ges 472-187. Springer, 2014.

[14] Joseph Redmon, Santosh Divvala, Ross Girshick, and Ali Farhadi ro nl look ce: Unified, real-time object detection. In Proceedings of the IEEE Conf rence on Sompu tr Vision and Pattern Recognition, pages 779-788, 2016.

[15] A. Kale, A. Sundaresan, A. N. Rajagopalan, N. P. Cun M, A. K. v-Chowdhury, V. Kruger, and R. Chellappa. Identification of humans using ga r. IEEE Transacy ns on Image Processing, 13(9):1163-1173, 2004.

[16] Ibrahim Venkat and Philippe De Wilde. Robust gan ognitio by learning and exploiting subgait characteristics. International Journal computer nwon, 91(1):7-23, 2011.

[17] Chiraz BenAbdelkader, Ross Cutler, Harsh Va da, a Larry Davis. Eigengait: Motion-based recognition of people using imao f-simila ity. In Audio-and Video-Based Biometric Person Authentication, pages 284-29 Spri ger.

[18] S. Sarkar, P. J. Phillips r. veya, P. Grother, and K. W. Bowyer. The humanid gait challenge problem: do ets, perfo noe, and analysis. IEEE Transactions on Pattern Analysis and Machine Intel genc 7(2):162-177, 2005.

[19] C. BenAbdel ader R. Cutler, d L. Davis. Stride and cadence as a biometric in automatic person identif tion a verification. In Automatic Face and Gesture Recognition, 2002. Proceedings. Fifth Is nternal nal Conference on, pages 372-377.

[20] $\mathrm{H}$ onos Fujiyos ald Alan J Lipton. Real-time human motion analysis by image skeletonizaa. In mosedings of the 4th IEEE Workshop on Applications of Computer Vision (WACV'98), pago IEEE Computer Society, 1998.

[21] S. A. Niy gi and E. H. Adelson. Analyzing and recognizing walking figures in xyt. In Computer Vision and Pattern Recognition, 1994. Proceedings CVPR '94., 1994 IEEE Computer Society Conference on, pages 469-474.

[22] Bridget Burdett. Measuring accessible journeys. Report, Traffic Design Group, July 2013.

[23] Casia gait database, http://www.cbsr.ia.ac.cn/english/gait\%20databases.asp.

[24] Zhe Cao, Tomas Simon, Shih-En Wei, and Yaser Sheikh. Realtime multi-person 2d pose estimation using part affinity fields. arXiv preprint arXiv:1611.08050, 2016. 